\title{
On the Feasibility of a Matched-Field Inversion in a Three-Dimensional Oceanic Environment Ignoring Out-of-Plane Propagation
}

\author{
Alexios Korakas, Member, IEEE, and Frédéric Sturm
}

\begin{abstract}
Inverse problems in ocean acoustics are based on 2-D modeling of sound propagation, hence ignoring the effects of horizontal refraction, referred to as 3-D propagation effects. However, the acoustic propagation in shallow-water environments, such as the continental shelf, may be affected by 3-D effects requiring 3-D modeling to be accounted for. The aim of this work is to investigate the importance of the 3-D effects with respect to the performance and reliability of typical 2-D-model-based inversion procedures of ocean acoustics. The study is carried out on a well-established synthetic test case which exhibits well-known 3-D effects. A matched-field inversion procedure is implemented based on the exhaustive search over the parameter space. The feasibility and the limits of inverting low-frequency noisy 3-D synthetic data for some parameters describing the test case by matching replica from 2-D computations are explored. Both synthetic data and replica are generated using a parabolic-equation-based code. This approach highlights the relevance of using 2-D propagation models when inversions are performed at relatively short ranges from the source. On the other hand, important mismatch occurs when inverting at farther ranges, demonstrating that the use of fully 3-D forward models is required.
\end{abstract}

Index Terms-Inversion, mismatch, shallow water, underwater acoustics, 3-D.

\section{INTRODUCTION}

$\mathbf{T}$ HIS paper deals with the importance of the effects of out-of-plane acoustic propagation with respect to the reliability and performance of inversion procedures typically applied in ocean acoustics (e.g., ocean acoustic tomography or geoacoustic inversions). In particular, we focus on shallow-water environments where the interaction of sound with the ocean bottom dominates acoustic propagation. Realistic shallow-water environments, like those encountered on the continental shelf, rarely present a horizontal bathymetry. Out-of-plane propagation effects, also referred to as 3-D effects, may thus occur from the successive reflections of the sound off the nonhorizontal seabed, during which the acoustic waves are gradually refracted in the horizontal direction [1]-[5].

Matched-field techniques for the inversion of recoverable parameters of the oceanic environment are particularly adapted

Manuscript received February 19, 2011; accepted May 18, 2011. Date of publication August 12, 2011; date of current version October 21, 2011.

Associate Editor: R. Chapman.

The authors are with the Laboratoire de Mécanique des Fluides et d'Acoustique (LMFA, UMR CNRS 5509-ECL-UCBL1-INSA Lyon), Ecole Centrale de Lyon, 69134 Ecully Cedex, France (e-mail: a.korakas@ gmail.com; frederic. sturm@ec-lyon.f)

Digital Object Identifier 10.1109/JOE.2011.2157730 to shallow-water environments and their use has become widespread over the last decades [6], [7]. Matched-field inversion relies on correlations between acoustic propagation field data measured on one or more hydrophone arrays, and replica of the acoustic field generated by an ocean acoustic propagation model, namely the forward model. The correlations are expressed through some cost function, and the inverse problem is formulated as an optimization process over a set of parameters involved in the forward model to obtain an estimation of their values.

It is clear that the performance and reliability of the above inversion procedure strongly depends on the forward model, and, more specifically, on the relevance of the simplifying physical assumptions underlying it. The modeling assumptions generally result from a tradeoff between speed and accuracy requirements. On the one hand, the forward model has to be fast enough for the inversions to be performed in reasonable times and, on the other hand, it has to take the important physical phenomena into account to correctly predict and interpret the propagation effects within some acceptable margin of error.

The most commonly used assumption until now is that of axially symmetric environments leading to the so-called 2-D models, i.e., models assuming that the sound energy is conserved within the vertical plane containing the source and the receiving array. Their main advantage is that they are fast and efficient, especially at low frequencies, in the sense that they lead to fast inversion procedures while providing sufficient accuracy to meet the needs of the considered applications.

Recently, a growing number of publications related to ocean acoustics put their focus on the continental shelf environment (see, for instance, [8]-[10]) which presents a relatively weakly sloping bathymetry. It is well known that acoustic propagation over a sloping bottom geometry is subject to horizontal refraction [1]-[5]. In this case, it has been demonstrated that the 2-D approach to propagation, which ignores out-of-plane propagation, fails to interpret the propagation effects and, instead, fully 3-D modeling is required to correctly predict the acoustic field [3], [11]-[16]. Nevertheless, no systematic study has been reported, to the authors' knowledge, dealing with the potential impact of out-of-plane propagation effects onto inversion results. The main reason lies in the significantly increased central processing unit (CPU) time requirements of fully 3-D models to such an extent that a 3-D-model-based inversion quickly becomes prohibitive for more than two parameters at a time. 
In a recent work [17], inversion results of acoustic data collected in a continental shelf area near the Florida coast [18] suggested that an important mismatch might occur when 2-D propagation is assumed, i.e., when out-of-plane propagation effects are ignored [19]. Clearly, the question that arises is whether the 3-D effects that might be present in the acoustic data measured in a continental shelf area can affect the performance and reliability of an inversion based on a 2-D forward model, and, in case the answer is yes, to which extent.

In this work, the feasibility and the limits of an inversion in a 3-D synthetic oceanic environment assuming 2-D propagation are investigated. The study is carried out on the 3-D extension of the Acoustical Society of America (ASA) wedge benchmark [20], as defined in [14], which is a well-established test case with well-known 3-D effects. A simple matched-field inversion procedure implemented in a Bayesian framework and based on the exhaustive search of the parameter space is used. It is briefly summarized in Section II. Although this approach is time consuming, it is robust and convergence check is straightforward. In Section III, the 3-D effects in the 3-D ASA wedge are recalled and the slope effect on inversion is examined by means of a sensitivity analysis of the objective function. This preliminary analysis aims at dictating us an efficient approach to deal with our problem but also aims at providing physical insight into the inversion results. The inversion results are presented and interpreted in Section IV. This is followed by a discussion concluding this paper. Efforts have been made to maintain a simple approach providing valuable insight without loss of generality.

\section{MATCHED-FIELD INVERSION APPROACH}

The problem of inverting acoustic field data due to a point source to infer parameters characterizing the oceanic environment is nonlinear due to the nonlinear relationship between the acoustic field and the parameters. The Bayesian approach to inversion thus offers a favorable framework for a meaningful interpretation of the solution including uncertainty analysis. When applied to ocean acoustics, this approach leads to the matched-field inversion technique [21], [22]. This section briefly describes the Bayesian formulation of the inverse problem and the specific approach used in this study. For further details, the reader is referred to standard texts on the matter, e.g., [23] and [24].

In the Bayesian formulation, the solution of the inverse problem is fully characterized by the posterior probability density (PPD) function of the parameters. Let the $N$-length complex vector $\mathbf{d}_{\text {obs }}$ denote the observed (or measured) acoustic pressure field data on an $N$-element receiving array at some location in the water column, and $\mathbf{d}(\mathbf{m})$ the predicted data obtained with an ocean acoustic propagation model as a function of the $M$-length vector $\mathbf{m}$ denoting the set of parameters to be recovered. The starting point of the formulation is the relation between the observed and the predicted data, which can be written as follows:

$$
\mathbf{d}_{\mathrm{obs}}=\mathbf{d}(\mathbf{m})+\mathbf{n}_{D}
$$

where the complex vector $\mathbf{n}_{D}$ denotes the error (or noise) term including both experimental and theory errors [23].

The error term $\mathbf{n}_{D}$ is generally not known and the assumption of complex Gaussian distributed errors is commonly adopted. Under this assumption, the PPD, defined as the probability density function of $\mathbf{m}$ given $\mathbf{d}_{\text {obs }}$, takes the following form:

$$
\mathrm{p}\left(\mathbf{m} \mid \mathbf{d}_{\mathrm{obs}}\right)=\frac{\exp [-\phi(\mathbf{m})] \mathrm{p}(\mathbf{m})}{\int_{\mathcal{M}} \exp \left[-\phi\left(\mathbf{m}^{\prime}\right)\right] \mathrm{p}\left(\mathbf{m}^{\prime}\right) \mathrm{d} \mathbf{m}^{\prime}}
$$

where $\phi(\mathbf{m})$ is the objective function of the inverse problem, $\mathrm{p}(\mathbf{m})$ is a probability density function reflecting the a priori information on the parameters, and the integral in the denominator is evaluated over the parameter space $\mathcal{M}$ whose dimension is $M$. The objective function is given by

$$
\phi(\mathbf{m})=\frac{1}{2}\left[\mathbf{d}_{\mathrm{obs}}-\mathbf{d}(\mathbf{m})\right]^{*} \mathbf{C}_{D}^{-1}\left[\mathbf{d}_{\mathrm{obs}}-\mathbf{d}(\mathbf{m})\right]
$$

where the $N \times N$ matrix $\mathrm{C}_{D}$ is the error (or data) covariance matrix with diagonal elements representing the variance in the data and off-diagonal elements representing the error correlations between receiver pairs. In practice, the predicted data are provided by propagation codes that do not take the source strength into account. The predicted data can thus be written as $\mathbf{d}(\mathbf{m})=S_{f} \mathbf{w}(\mathbf{m})$, where $S_{f}$ is the complex source strength at frequency $f$, and $\mathbf{w}(\mathbf{m})$ denotes the replica vector of the acoustic field at the array computed by a propagation code at the same frequency.

The objective function can be expressed in various forms depending on the content of the data to be exploited in the inversion [25]. In this study, we consider a single-frequency source signal with unknown source strength. Additionally, assuming spatially uncorrelated errors along the array (i.e., from one receiver to another), the error covariance matrix writes $\mathbf{C}_{D}=$ $\nu_{D} \mathbf{I}$, where $\nu_{D}$ denotes the variance in the data and $\mathbf{I}$ the identity matrix. The objective function is thus obtained according to [21], [22], and [25] as

$$
\phi(\mathbf{m})=\mathcal{B}(\mathbf{m})\left\|\mathbf{d}_{\mathrm{obs}}\right\|^{2} / \nu_{D}
$$

where $\mathcal{B}(\mathbf{m})$ is the Bartlett function, correlating the observed data with the replica, given as

$$
\mathcal{B}(\mathbf{m})=1-\frac{\left|\mathbf{w}^{*}(\mathbf{m}) \mathbf{d}_{\text {obs }}\right|^{2}}{\|\mathbf{w}(\mathbf{m})\|^{2}\left\|\mathbf{d}_{\mathrm{obs}}\right\|^{2}} .
$$

In this form, the Bartlett processor values are confined between 0 and 1 , where 0 corresponds to a perfect match between the observed data and the replica. Note here that, whereas $\nu_{D}$ and $\left\|\mathbf{d}_{\text {obs }}\right\|$ in (4) depend on frequency, they are considered constant in a single-frequency inversion.

The PPD is a multidimensional function for $M \geqslant 2$ and various statistical measures can be introduced for its interpretation. We here resort to the posterior mean estimate 
$\boldsymbol{\mu}=\left[\mu_{1}, \ldots, \mu_{M}\right]^{T}$ of the parameter set, where the mean estimate $\mu_{i}$ of the $i$ th parameter is defined as $\mu_{i}=\mathrm{E}\left[m_{i}\right]$ with

$$
\mathrm{E}\left[m_{i}\right]=\int_{\mathcal{M}} m_{i}^{\prime} \mathrm{p}\left(\mathbf{m}^{\prime} \mid \mathbf{d}_{\mathrm{obs}}\right) \mathrm{d} \mathbf{m}^{\prime} .
$$

The standard deviations $\sigma_{i}=\sqrt{\operatorname{var}\left(m_{i}\right)}, 1 \leqslant i \leqslant M$, are used for the assessment of the dispersion of the estimate, where $\operatorname{var}\left(m_{i}\right)$ denotes the variance of the $i$ th parameter defined as

$$
\operatorname{var}\left(m_{i}\right)=\mathrm{E}\left[\left(m_{i}-\mu_{i}\right)^{2}\right]
$$

Since the relationship between the data and the parameters is nonlinear, the PPD does not necessarily follow a Gaussian distribution. The mean estimate and its standard deviation for the interpretation of the solution should thus be used with care. In particular, it is useful to visualize the posterior marginal densities of each parameter, obtained by integrating $M-1$ times the PPD as below

$$
\mathrm{p}\left(m_{i} \mid \mathbf{d}_{\mathrm{obs}}\right)=\int_{\mathcal{M}} \delta\left(m_{i}^{\prime}-m_{i}\right) \mathrm{p}\left(\mathbf{m}^{\prime} \mid \mathbf{d}_{\mathrm{obs}}\right) \mathrm{d} \mathbf{m}^{\prime} .
$$

The mean estimate and standard deviation thus represent acceptable measures for a parameter whenever its posterior marginal density is unimodal and quasi-symmetric.

In practice, many authors resort to the maximum a posteriori (MAP) estimate providing the best match between the observed data and the replica (e.g., see [6]). The MAP estimate denoted $\mathbf{m}_{\mathrm{MAP}}$ is defined as the parameter vector that maximizes the PPD

$$
\mathrm{p}\left(\mathbf{m}_{\mathrm{MAP}} \mid \mathbf{d}_{\mathrm{obs}}\right)=\max _{\mathbf{m} \in \mathcal{M}}\left\{\mathrm{p}\left(\mathbf{m} \mid \mathbf{d}_{\mathrm{obs}}\right)\right\}
$$

or, equivalently, that minimizes the objective function

$$
\phi\left(\mathbf{m}_{\mathrm{MAP}}\right)=\min _{\mathbf{m} \in \mathcal{M}}\{\phi(\mathbf{m})\} .
$$

Since $\nu_{D}$ and $\left\|\mathbf{d}_{\mathrm{obs}}\right\|$ are constant in (4), this is also equivalent to minimizing the Bartlett function. Its value at the MAP estimate $\mathcal{B}\left(\mathbf{m}_{\mathrm{MAP}}\right)$ will be considered here to assess the match between the observed data and the replica. To this end, it is also useful to visualize the comparison of the transmission loss (TL) curves along the array corresponding to the observed data with those corresponding to the replica obtained using the MAP estimate. Note finally that obtaining the MAP estimate does not require the computation of the $M$-dimensional integral in the denominator of (2). However, it does not provide any indication on the dispersion of the estimate.

The evaluation of the PPD and the measures (6)-(8) for its interpretation requires the evaluation of $M$-dimensional integrals where $M$ can generally be large. To address this issue, many authors consider the use of elaborate optimization algorithms [21], [22], [24] to efficiently sample the parameter space. However, for the purpose of this work, the PPD is sampled over the parameter space using exhaustive search, also referred to as grid search [24]. Although time consuming, this approach is known

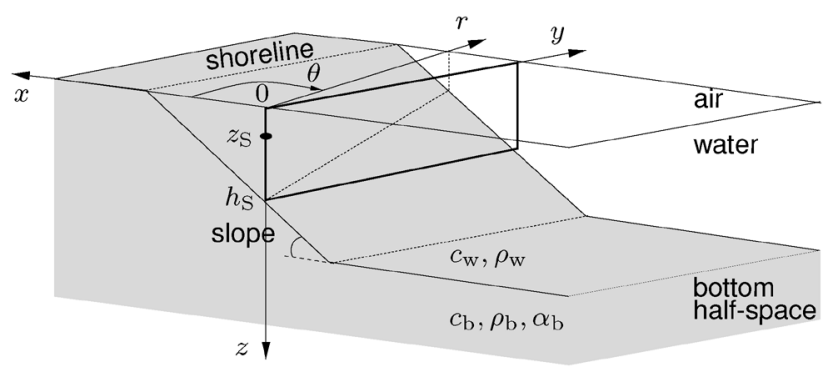

Fig. 1. Schematic representation of the 3-D ASA wedge benchmark case.

to be robust and convergence check is straightforward. In practice, the exhaustive search requires defining bounds for each parameter and discretizing the resulting intervals. Convergence is obtained by sufficiently refining the discretization of the parameter space.

\section{SYNTHETIC ENVIRONMENT: A PRELIMINARY ANALYSIS}

The synthetic environment considered in this study is the 3-D ASA wedge benchmark shown in Fig. 1. It consists of a water layer lying over a fluid bottom half-space with a constant slope of $5 \%\left(\approx 2.86^{\circ}\right)$ in the $x$-direction (or, equivalently, for $\theta=0^{\circ}$ ). The water depth $h_{\mathrm{S}}$ at the source is $200 \mathrm{~m}$, the water sound speed $c_{\mathrm{W}}$ is $1500 \mathrm{~m} / \mathrm{s}$, and the water density $\rho_{\mathrm{w}}$ is $1 \mathrm{~g} / \mathrm{cm}^{3}$. The bottom sound speed $c_{\mathrm{b}}$ is equal to $1700 \mathrm{~m} / \mathrm{s}$, the bottom density $\rho_{\mathrm{b}}$ to $1.5 \mathrm{~g} / \mathrm{cm}^{3}$, and the attenuation of sound $\alpha_{\mathrm{b}}$ in the bottom to $0.5 \mathrm{~dB} / \lambda$. A point source is positioned at a depth $z_{\mathrm{S}}$ of $40 \mathrm{~m}$. We focus here on the vertical plane oriented in the across-slope $y$-direction (or, equivalently, in the azimuthal direction corresponding to $\theta=90^{\circ}$ ) along which the water depth is constant and equal to $h_{\mathrm{S}}$ (see thick line rectangle in Fig. 1).

The acoustic field along a receiving array positioned in the across-slope direction is known to be highly 3-D, i.e., it is affected by out-of-plane propagation resulting in strong 3-D effects, such as multiple mode arrivals, mode shadow zones, and intramode interference phenomena. The 3-D effects are interpreted as the accumulated effect of the multiple interactions of sound with the sloping interface during which the sound is gradually horizontally refracted towards the downslope direction [1]-[5]. From the viewpoint of propagation modeling, the 3-D aspect of the field is determined by one single parameter: the slope. Therefore, to investigate the impact of the 3-D effects onto inversion results, we need to examine, in a preliminary step, the sensitivity of the objective function (4) with respect to the slope value. The sensitivity analysis also permits to identify the most important parameters with respect to the inverse problem.

Although the parameters are constant within the vertical plane across slope, the field presents gradual and abrupt variations as a function of range along that specific azimuthal direction. Several array ranges are thus examined. In what follows, the 3-D effects predicted for the 3-D ASA wedge are first described. The frequencies of 25 and $35 \mathrm{~Hz}$ are considered, for which three and four propagating modes are excited at the source, respectively. The sensitivity analysis is then performed on both horizontal line arrays (HLAs) and vertical line arrays (VLAs) considering several array ranges chosen accordingly. 


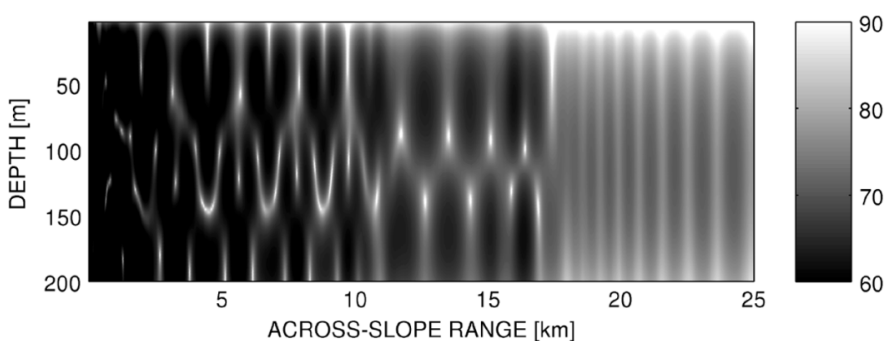

Fig. 2. Transmission loss ( $\mathrm{dB}$ re $1 \mathrm{~m})$ in the vertical plane across slope due to a $25-\mathrm{Hz}$ point source located at a depth of $40 \mathrm{~m}$.

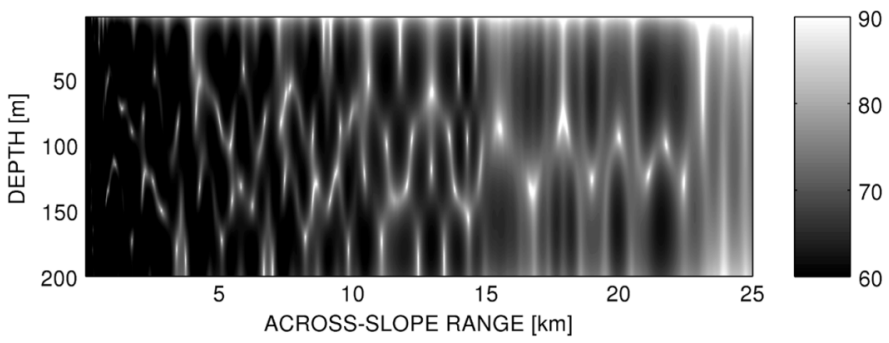

Fig. 3. Transmission loss $(\mathrm{dB}$ re $1 \mathrm{~m})$ in the vertical plane across slope due to a $35-\mathrm{Hz}$ point source located at a depth of $40 \mathrm{~m}$.

TABLE I

TyPICAL 3-D EFFECTS FOR THE 3-D ASA WedGe TeSt CASE OBSERVED IN THE ACROSS-SLOPE DIRECTION AS A FUNCTION OF RANGE FROM THE SOURCE

\begin{tabular}{|c|c|c|c|c|}
\hline & Mode 1 & Mode 2 & Mode 3 & Mode 4 \\
\hline$f=25 \mathrm{~Hz}$ & \multicolumn{4}{|c|}{} \\
\hline Second arrival & $\gtrsim 16 \mathrm{~km}$ & $\approx 14.5 \mathrm{~km}$ & none & - \\
\hline Cut-off range & $>25 \mathrm{~km}$ & $\approx 17.5 \mathrm{~km}$ & $\approx 11 \mathrm{~km}$ & - \\
\hline$f=35 \mathrm{~Hz}$ & \multicolumn{5}{|c|}{} \\
\hline Second arrival & $\gtrsim 17 \mathrm{~km}$ & $\approx 16 \mathrm{~km}$ & $\gtrsim 12 \mathrm{~km}$ & none \\
\hline Cut-off range & $>25 \mathrm{~km}$ & $\approx 23 \mathrm{~km}$ & $\approx 15 \mathrm{~km}$ & $\approx 11 \mathrm{~km}$ \\
\hline
\end{tabular}

\section{A. Description of the 3-D Effects}

Figs. 2 and 3 show the TL versus depth and across-slope range as predicted by the fully 3-D parabolic equation (PE)-based code 3DWAPE [15] for the 3-D ASA wedge and for the frequencies of 25 and $35 \mathrm{~Hz}$, respectively. By inspecting the interference fringes in each figure, several zones can be identified as a function of range, each zone being associated to different modal contributions in the acoustic field. These are described below for each frequency. Typical 3-D effects such as multiple mode arrivals and mode shadow zones for each of the two frequencies are summarized in Table I.

At the frequency of $25 \mathrm{~Hz}$, three propagating modes are excited at the source. Three zones can be identified as a function of range. The first zone, extending up to approximately $11 \mathrm{~km}$, is characterized by single arrivals of each mode initially present at the source. Note that the interference fringes tend to shrink with increasing range in the across-slope direction. At the range of approximately $11 \mathrm{~km}$, mode 3 reaches its cutoff range. The second zone extends then up to $\approx 17.5 \mathrm{~km}$ corresponding to the cutoff range of mode 2 . This zone exhibits typical interference

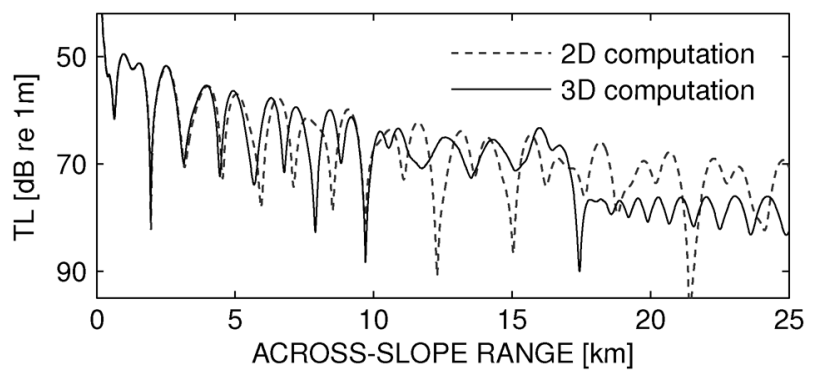

Fig. 4. Comparison of TL versus across-slope range corresponding to 2-D (dashed line) and 3-D (solid line) computations at the frequency of $25 \mathrm{~Hz}$ for a receiver depth of $40 \mathrm{~m}$.

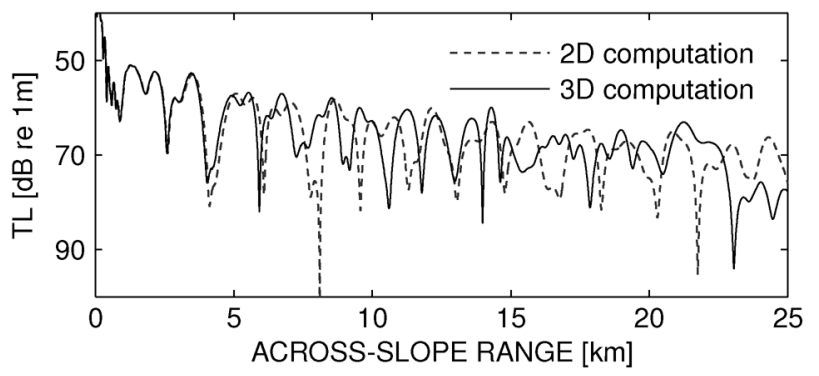

Fig. 5. Comparison of TL versus across-slope range corresponding to 2-D (dashed line) and 3-D (solid line) computations at the frequency of $35 \mathrm{~Hz}$ for a receiver depth of $40 \mathrm{~m}$.

patterns between modes 1 and 2 . Note here that by initializing the PE marching algorithm by each of the three modes individually (as in [14]-[16]), one can show that beyond the ranges of 16 and $14.5 \mathrm{~km}$, second arrivals of, respectively, mode 1 and 2 contribute to the field. The increase in the field intensity observed at the range of approximately $17 \mathrm{~km}$ is due to a caustic effect between the two arrivals of mode 2 . Finally, the third zone extends beyond the range of $17.5 \mathrm{~km}$. This zone is characterized by intramode interference effects between the two distinct arrivals of mode 1 . (Mode 1 eventually enters its shadow zone at a range larger than $25 \mathrm{~km}$.)

At the frequency of $35 \mathrm{~Hz}$, four propagating modes are excited at the source. Again, propagating each single mode individually allows us to identify four zones in the across-slope direction. The first zone extends from the source up to approximately $11 \mathrm{~km}$ corresponding to the cutoff range of mode 4 . As before, this zone is characterized by single arrivals of each of the four modes. In the second zone, extending from $11 \mathrm{~km}$ up to $\approx 15 \mathrm{~km}$, typical interference fringes between modes 1,2 , and 3 are observed with a second arrival of mode 3 also contributing beyond the range of $12 \mathrm{~km}$. Mode 3 cutoff occurs at the range of $\approx 15 \mathrm{~km}$. The third zone, from the latter range up to $\approx 23 \mathrm{~km}$ where mode 2 enters its shadow zone, includes contributions from modes 1 and 2 with second arrivals of these two modes occurring beyond 16 and $17 \mathrm{~km}$, respectively. The caustic effect now occurs for both modes 3 and 2 just before their cutoff at the respective ranges of 14 and $21.5 \mathrm{~km}$. Finally, the fourth zone extending beyond the range of $23 \mathrm{~km}$ exhibits interference fringes between the two arrivals of mode 1 . (Mode 1 is eventually cut off at a range far beyond $25 \mathrm{~km}$.)

The above description will be useful when interpreting the results in the remainder of this paper. Before proceeding, 

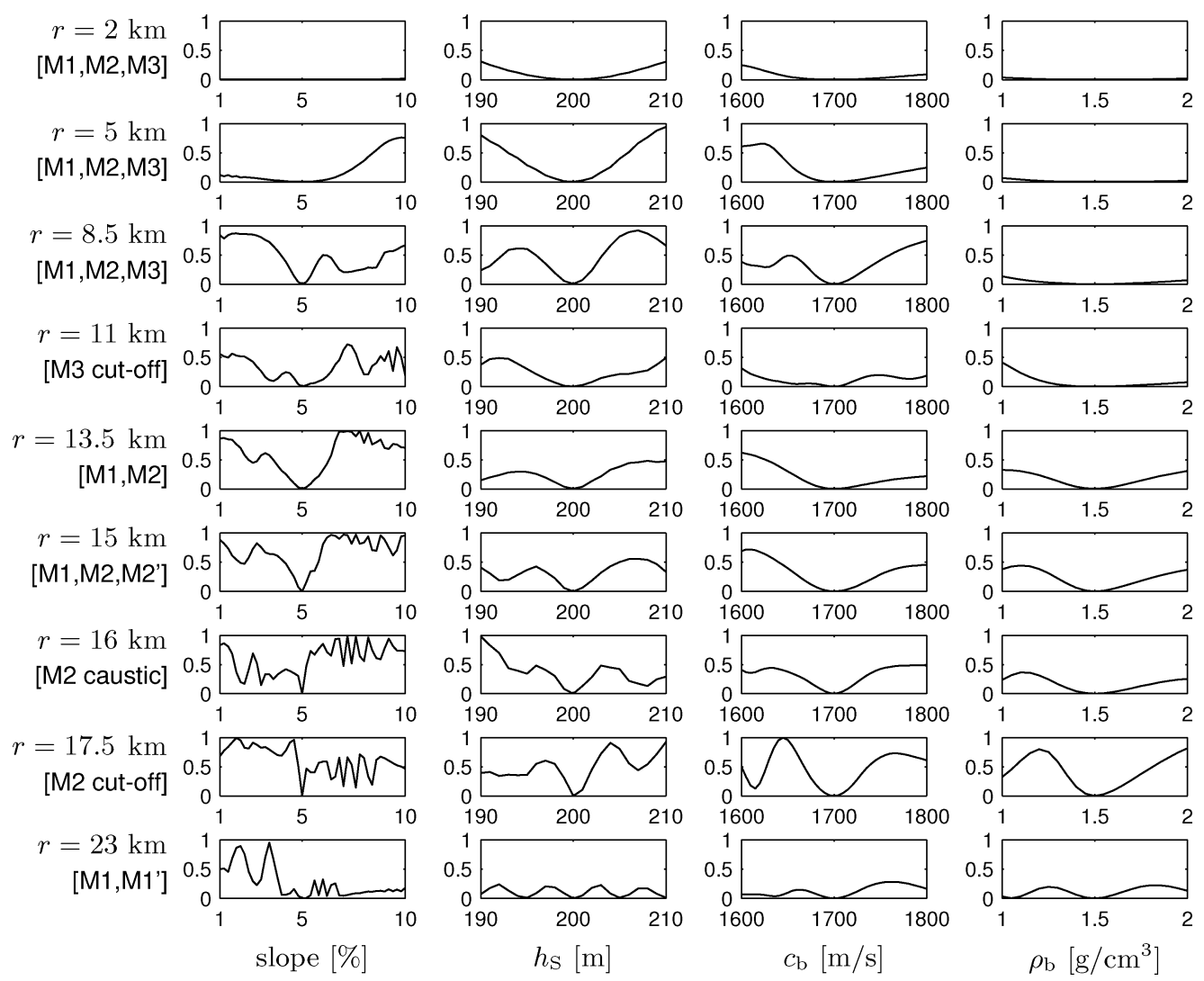

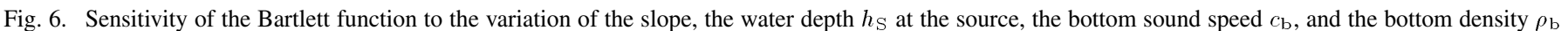

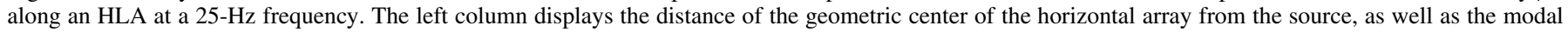

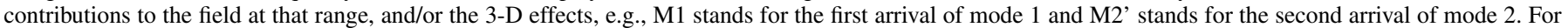
each parameter, the middle value displayed on the abscissa denotes its true value.

let us compare the curves of TL versus across-slope range obtained with a fully $3-\mathrm{D}$ computation to those obtained with a 2-D computation, i.e., ignoring horizontal refraction. The comparisons are shown in Figs. 4 and 5 for the respective frequencies of 25 and $35 \mathrm{~Hz}$ and for a receiver depth of $40 \mathrm{~m}$. Despite the evident differences in the 2-D and 3-D computations at long ranges, pointing out the need for 3-D modeling to correctly predict the field, we focus here on the similarities in the TL curves up to the distance of approximately $5 \mathrm{~km}$. This preliminary observation suggests that an inversion based on 2-D propagation modeling might be feasible, at least at relatively short ranges.

\section{B. Slope Effect on Inversion: A Sensitivity Analysis}

The sensitivity analysis of the objective function is intended to provide a preliminary indication on the most important parameters (which are involved in the forward model) with respect to the inverse problem. The sensitivity of the objective function is examined by graphical inspection of its response to the variation of one parameter at a time over discrete values within predefined bounds reflecting the a priori information. For single-frequency inversions, this is equivalent to examining the Bartlett function's response as given in (5). Both the observed data and the replica are generated by means of fully 3 -D computations using the 3DWAPE code. In particular, the observed data, simulating the acoustic pressure field on an HLA or a VLA at some locations in the water column, are computed using the nominal (or true) parameter values for the 3-D ASA wedge test case, and a new replica vector is generated for each discrete value of the parameters.

Results obtained at a frequency of $25 \mathrm{~Hz}$ with an HLA are displayed in Fig. 6 for the dominant parameters, namely, from left to right, the slope, the water depth $h_{\mathrm{S}}$, the bottom sound speed $c_{\mathrm{b}}$, and the bottom density $\rho_{\mathrm{b}}$ (others not shown here). The horizontal array considered is $1 \mathrm{~km}$ long containing 21 equidistant receivers and it is immerged at the source depth $(40 \mathrm{~m})$ to avoid mode cancellation. The sensitivity of the Bartlett function is examined for several array ranges (from top to bottom in Fig. 6) corresponding to different modal contributions and/or specific 3-D effects (summarized in the left column of Fig. 6). Note that the array range corresponds to the range of its geometric center. The parameters are varied within bounds indicated in the respective abscissa in Fig. 6, with the middle values denoting the true ones. The true value, the intervals, and their discretization for each parameter are summarized in Table II. The discretization is sufficiently fine to sense the fluctuations in the Bartlett response.

In the first zone, up to the range of $8.5 \mathrm{~km}$, where single arrivals of all the modes initially excited at the source contribute to the field, the objective function becomes more sensitive to the variation of the parameter values with increasing range, for all the parameters except $\rho_{\mathrm{b}}$. In others words, the information contained in the data along the array becomes more important with increasing range for the respective parameters, as expected due 

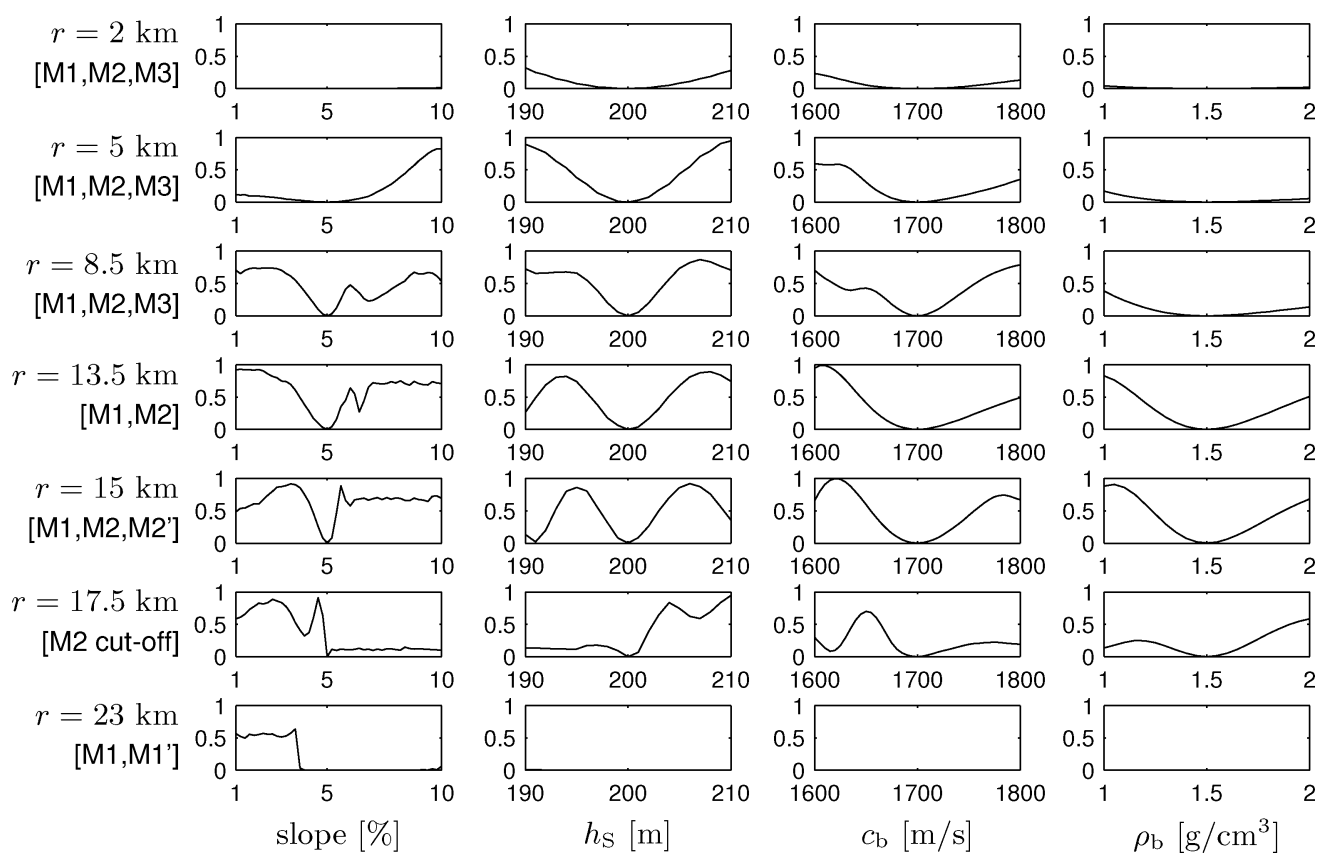

Fig. 7. Sensitivity of the Bartlett processor to the variation of the slope, the water depth $h_{\mathrm{S}}$ at the source, the bottom sound speed $c_{\mathrm{b}}$, and the bottom density $\rho_{\mathrm{b}}$ along a VLA at a $25-\mathrm{Hz}$ frequency. The left column displays the distance of the vertical array from the source, as well as the modal contributions to the field at that range, and/or the 3-D effects, e.g., M1 stands for the first arrival of mode 1 and M2' stands for the second arrival of mode 2. For each parameter, the middle value displayed on the abscissa denotes its true value.

TABLE II

TRUe VALUES, INTERVALS, AND DISCRETIZATION FOR THE PARAMETERS USED IN THE SENSITIVITY STUDY

\begin{tabular}{|c|c|c|c|c|}
\hline Parameter & True value & Bounds & Step & Samples \\
\hline slope $[\%]$ & 5.00 & {$[1.00,10.00]$} & 0.20 & 46 \\
\hline$h_{\mathrm{S}}[\mathrm{m}]$ & 200.00 & {$[190.00,210.00]$} & 1.00 & 21 \\
\hline$c_{\mathrm{b}}[\mathrm{m} / \mathrm{s}]$ & 1700.00 & {$[1600.00,1800.00]$} & 5.00 & 41 \\
\hline$\rho_{\mathrm{b}}\left[\mathrm{g} / \mathrm{cm}^{3}\right]$ & 1.50 & {$[1.00,2.00]$} & 0.05 & 21 \\
\hline
\end{tabular}

to the continuous interaction of sound with the water/bottom interface. It is important to note here that the response of the Bartlett function to the variation of the slope at the range of $2 \mathrm{~km}$ is flat, i.e., the slope effect at that range appears to be negligible. The trend observed up to the range of $8.5 \mathrm{~km}$ stops abruptly at 11 $\mathrm{km}$ for $h_{\mathrm{S}}, c_{\mathrm{b}}$, and $\rho_{\mathrm{b}}$ due to the cutoff of mode 3 corresponding to a loss of information in the data. Further, in the second zone, up to $17.5 \mathrm{~km}$ where mode 2 reaches its cutoff range, we observe an increase in the sensitivity of the objective function with range with respect to all parameters, with the slope being dominant. Note in particular the steep peak around the true value of the slope at 16 and $17.5 \mathrm{~km}$, corresponding respectively to the caustic and the cutoff of mode 2 , as well as the important improvement for the other parameters at the latter range. This can be attributed to the abrupt transition in the sound intensity between the second and third zones. Finally, at $23 \mathrm{~km}$, mode 2 is in its shadow zone yielding again a loss of information and the Barlett response becomes less sensitive.

We examine now the results of Fig. 7 obtained using a VLA for some of the ranges considered previously. The array used here is a 180 -m-long vertical array containing 19 equidistant receivers spanning the water column from 10- to 190-m depth. The trend observed up to $15 \mathrm{~km}$ is comparable to that of Fig. 6 . However, at $17.5 \mathrm{~km}$, the Bartlett function appears to be insen- sitive to the slope for higher values than the true one. Similar observations apply for the other parameters considered. Moreover, at $23 \mathrm{~km}$ (except for lower slope values than the true value), the Bartlett's response to the variation of the parameters is completely flat.

In both cases, and in addition to the 3-D versus 2-D comparisons shown above (see, for instance, Figs. 4 and 5), the almost flat Bartlett response to the variation of the slope at relatively short ranges (e.g., up to $5 \mathrm{~km}$ ) offers a preliminary indication that an inversion for the recovery of the other parameters based on 2-D propagation modeling might be successful, provided the slope is not coupled to them. The coupling can be examined by varying two parameters at a time and by evaluating the Bartlett function for each combination of them. Fig. 8 shows the result of this procedure for the slope and the water depth $h_{\mathrm{S}}$ considering, from left to right, HLAs positioned at 1,2 , and $5 \mathrm{~km}$ from the source. Note that, at each range, the computation of $46 \times 21=966$ replica of the 3-D acoustic pressure field was required, involving CPU times (on a single processor) ranging from $3 \mathrm{~h}$ for the range of $1 \mathrm{~km}$ to $15 \mathrm{~h}$ for the range of $5 \mathrm{~km}$. Fig. 8 gives evidence of a positive correlation between the slope and the water depth which appears negligible at $1 \mathrm{~km}$ [Fig. 8(a)] and becomes more important as range increases. In particular, we note that the coupling between the slope and the water depth at the range of $2 \mathrm{~km}$ [Fig. 8(b)] cannot be neglected. Consequently, an inversion at that range based on a 2-D model might lead to inconsistencies. Instead, an inversion using a line array positioned at $1 \mathrm{~km}$ from the source is more likely to succeed.

Let us now analyze the results at the frequency of 35 $\mathrm{Hz}$. The trend observed in the previous case is maintained 

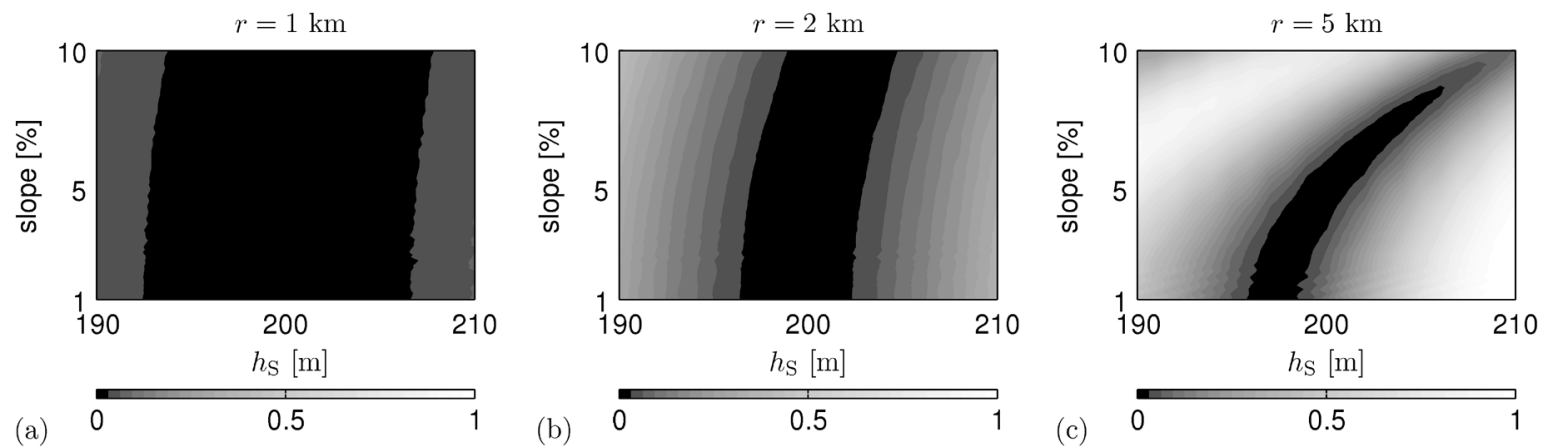

Fig. 8. Correlations between the slope and the water depth $h_{\mathrm{S}}$ at a $25-\mathrm{Hz}$ frequency considering a horizontal array at the ranges of (a) 1 $\mathrm{km}$, (b) $2 \mathrm{~km}$, and (c) $5 \mathrm{~km}$.

here in the first and second zones, up to $15 \mathrm{~km}$, despite the cutoff of mode 4 at $11 \mathrm{~km}$. Note again the steep peak in the Bartlett response around the true value of the slope due to the caustic and cutoff of mode 3 . At $16 \mathrm{~km}$, where mode 3 is in its shadow zone, the Bartlett function becomes less sensitive. Beyond that range, we observe an improvement, probably due to the second arrival of mode 2, which is continued up to the range of $23 \mathrm{~km}$. It is to be noticed again that near the caustic and cutoff of mode 2 the Bartlett function becomes highly sensitive around the true values of the slope, and to a lesser extent for the other parameters as well. Beyond the cutoff of mode 2, the sensitivity of the Barlett response decreases as can be observed at the range of $24 \mathrm{~km}$.

\section{MATCHED-FIELD INVERSION USING 2-D REPLICA}

The previous section suggests that, for a given environment, the feasibility of inverting acoustic field data over a sloping bottom ignoring out-of-plane propagation depends on the source-array separation. In this section, we perform a matched-field inversion based on a 2-D forward model for the recovery of the important parameters of the 3-D ASA wedge (Fig. 1) at the frequencies of 25 and $35 \mathrm{~Hz}$, considering several array ranges chosen according to the above analysis. It is understood that this approach does not permit the inversion for the slope value. Rather, the scenario simulated here is that of an inversion for the recovery of the bottom parameters $c_{\mathrm{b}}$ and $\rho_{\mathrm{b}}$ with uncertain knowledge of the water depth $h_{\mathrm{S}}$. The other parameters are kept fixed at their nominal values (see Section III).

Synthetic data are generated by means of fully 3-D computations using the 3DWAPE code, and zero-mean complex Gaussian-distributed white noise is added with a variance yielding a signal-to-noise ratio of $20 \mathrm{~dB}$. The data are computed on a $180-\mathrm{m}$-long VLA containing 19 equidistant receivers spanning the water column from 10- to 190-m depth. The replica are generated on the same array using the same code by means, however, of 2-D computations, i.e., neglecting out-of-plane propagation. Note that the numerical parameters of the PE-based code used in both cases are identical for the use of the objective function given in (4) to be valid (since unknown modeling errors are avoided). The true values of $c_{\mathrm{b}}$, $\rho_{\mathrm{b}}$, and $h_{\mathrm{S}}$, the search intervals and their discretization used in the inversion process are similar to those given in Table II. The a priori probability density is considered here uniform over the parameter space and the PPD is sampled using exhaustive search.

Let us first analyze the results obtained with vertical arrays positioned up to $10 \mathrm{~km}$ from the source. For both frequencies, this area is characterized by single arrivals of each mode initially present at the source (see Figs. 2 and 3 and Table I). The results at the frequencies of 25 and $35 \mathrm{~Hz}$ are shown, respectively, in Figs. 10 and 11. Each figure has three subfigures corresponding to vertical arrays positioned at (a) $1 \mathrm{~km}$ where, according to the previous analysis, the effect of the slope is not important, (b) 5 $\mathrm{km}$, and (c) $8.5 \mathrm{~km}$ where the slope effect becomes more important as range increases. In each subfigure, the left panel shows the posterior marginal densities for each parameter. The vertical dotted line corresponds to the true value of each parameter, the circle represents the MAP estimate, and the cross with the horizontal line represents the mean estimate and its standard deviation. The right panel shows comparisons of the TL curves along the vertical array corresponding to the 3-D synthetic data (dashed line), i.e., for the nominal values of the parameters, with those corresponding to 2-D replica computed using the MAP estimate (solid line). The Bartlett values at the MAP estimate are given in Table III.

At the range of $1 \mathrm{~km}$ and for both frequencies [Figs. 10(a) and 11(a)], the correct values of the parameters are retrieved with a satisfactory accuracy in the estimates. An excellent match between synthetic data and replica is obtained for the MAP estimate (see Table III) and an almost perfect superposition of the TL curves is observed. Note that the nonzero value of the Bartlett function is mainly due to the white noise in the data. This result thus suggests that the effect of the slope at $1 \mathrm{~km}$ can be neglected and an inversion assuming 2-D propagation is successful. At the range of $5 \mathrm{~km}$ [Figs. 10(b) and 11(b)], the marginal densities indicate that the estimates are obtained with a good accuracy, however, the retrieved values turn out to be biased. In addition, the TL comparisons are still excellent and the Bartlett values at the MAP estimate is unchanged. In other words, the result at the range of 5 $\mathrm{km}$ is a typical mismatch case [26] where, in addition, we have no indication that the estimates are erroneous. In the context of real data applications, in which the true values 

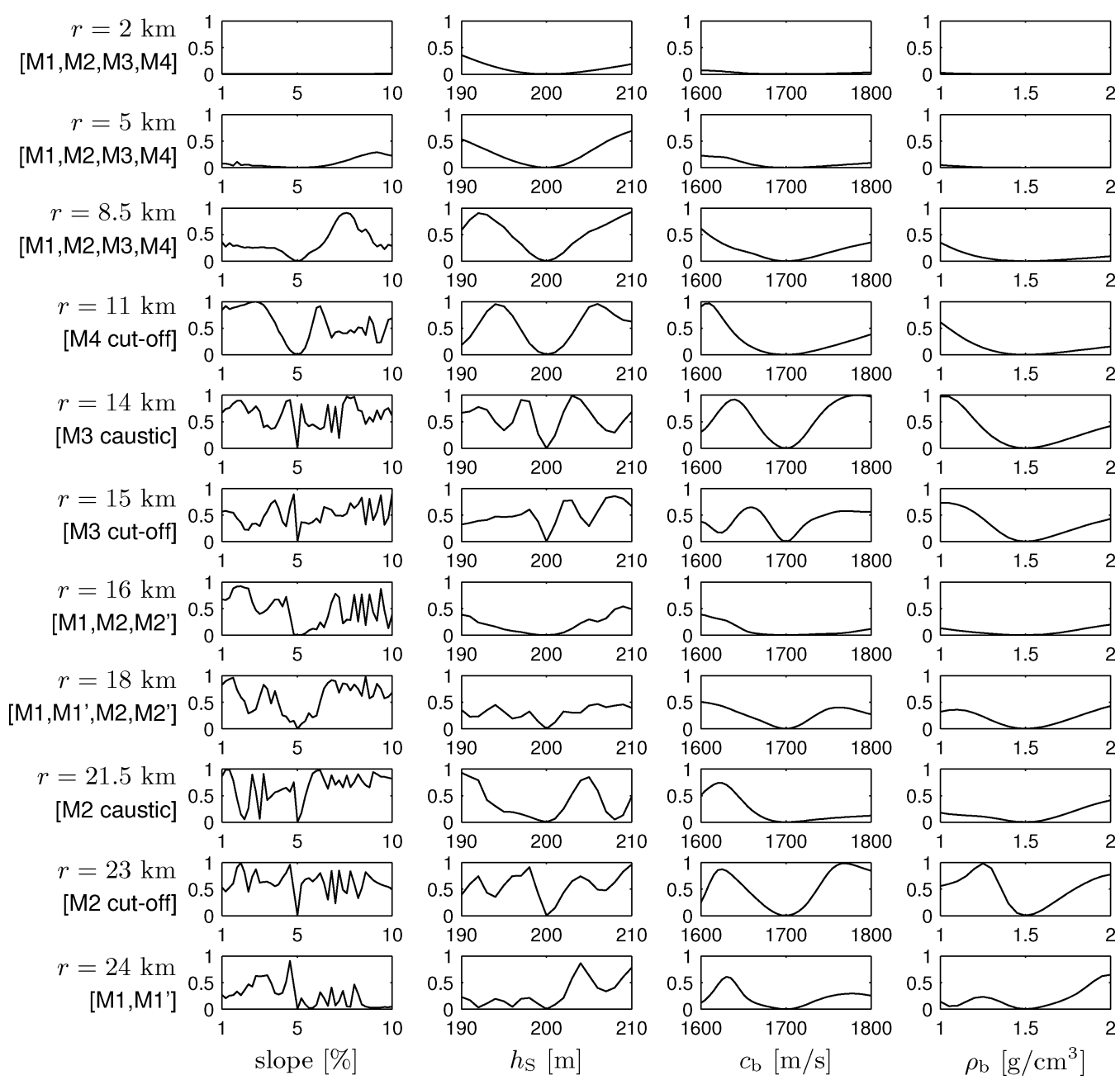

Fig. 9. Sensitivity of the Bartlett processor to the variation of the slope, the water depth $h_{\mathrm{S}}$ at the source, the bottom sound speed $c_{\mathrm{b}}$, and the bottom density $\rho_{\mathrm{b}}$ along an HLA at a $35-\mathrm{Hz}$ frequency. The left column displays the distance of the geometric center of the horizontal array from the source, as well as the modal contributions to the field at that range, and/or the 3-D effects, e.g., M1 stands for the first arrival of mode 1 and M2' stands for the second arrival of mode 2. For each parameter, the middle value displayed on the abscissa denotes its true value.

of the parameters are not known before the inversion, the risk of erroneous estimation is potentially important.

This behavior can be explained as follows. Recall that, although single mode arrivals contribute to the field at the range of $5 \mathrm{~km}$, these are 3-D arrivals and not direct 2-D arrivals. In other words, these arrivals do not correspond to acoustic waves that traveled within the vertical plane containing the source and the receiving array but, rather, they followed hyperbolic paths (as viewed from above), i.e., they correspond to waves that propagated out from the source in the up-slope direction and were gradually refracted downslope before reaching the receiver. As a result, these 3-D arrivals traveled a longer distance and through shallower waters than the direct 2-D arrivals. Therefore, the inversion process tends to adapt the parameter values in the 2-D forward model to account for the effects of horizontal refraction at the range of $5 \mathrm{~km}$. Since single mode arrivals alone contribute to the field at that range, the 2-D forward model succeeds in compensating for the 3-D propagation effects. A similar behavior is observed for an array located at the range of $8.5 \mathrm{~km}$ [Figs. 10(c) and 11(c)], although slightly exaggerated due to the stronger 3-D effects at that range. Note here, however, that the
TL comparisons and the Bartlett values at the MAP estimate may suggest that the retrieved parameters are potentially erroneous.

Let us now examine the respective results of Figs. 12 and 13 for arrays located at (a) $13.5 \mathrm{~km}$, (b) $16 \mathrm{~km}$, and (c) 19 $\mathrm{km}$, where typical 3-D effects occur for both frequencies, including mode shadow zones and second mode arrivals. These can be easily identified in Figs. 2 and 3 (see also Table I). The 2-D-model-based inversion process now tends to adapt the parameter values so as to additionally compensate for the reduced (and range-dependent) number of modes contributing to the field at the respective ranges. For instance, this can be clearly seen at the frequency of $25 \mathrm{~Hz}$ for the range of 19 $\mathrm{km}$ [Fig. 12(c)] by inspecting the TL curves corresponding to the 3-D synthetic data and to the 2-D replica computed using the MAP estimate. Recall that, at the frequency of 25 $\mathrm{Hz}$, three modes are excited at the source. Recall also that the receiving array at the range of $19 \mathrm{~km}$ is in the shadow zone of modes 2 and 3 , and two arrivals of mode 1 alone contribute to the field. Accordingly, the TL curve corresponding to the 3-D synthetic data [dashed line in Fig. 12(c)] has no nodes. 

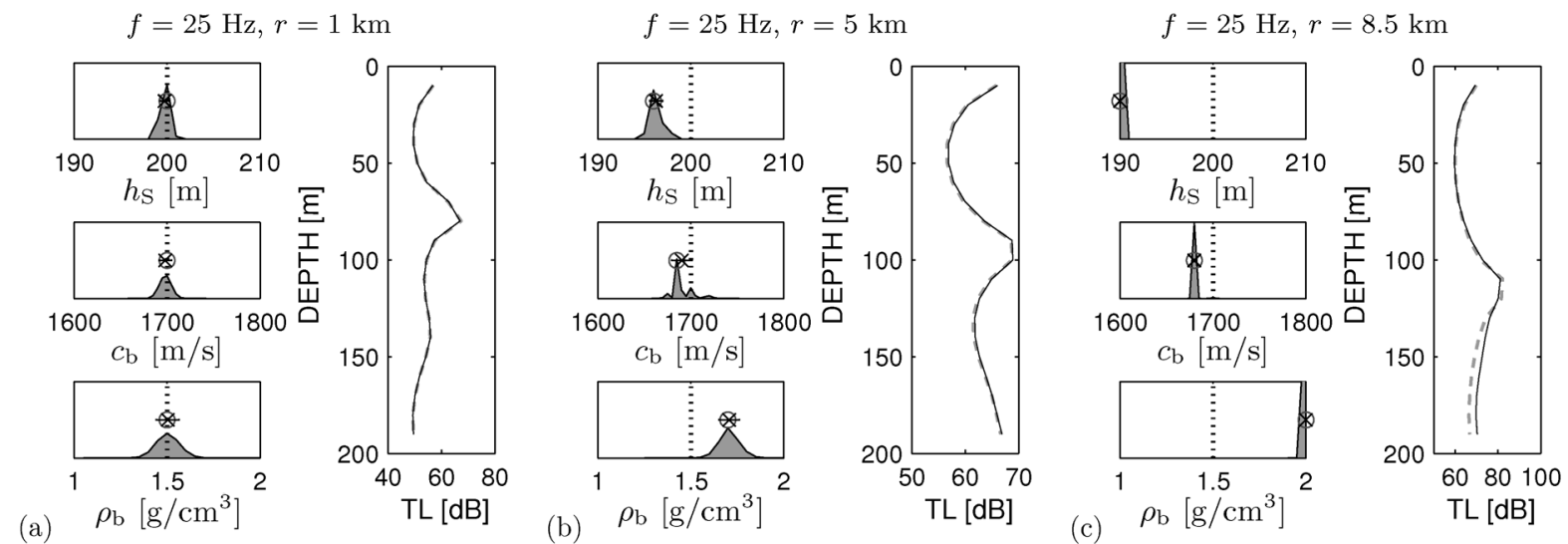

Fig. 10. Left panel of each subfigure: marginal posterior densities of $h_{\mathrm{S}}, c_{\mathrm{b}}$, and $\rho_{\mathrm{b}}$ for a 25-Hz frequency inversion assuming 2-D propagation along a vertical array located at (a) $1 \mathrm{~km}$, (b) $5 \mathrm{~km}$, and (c) $8.5 \mathrm{~km}$. The vertical dotted line represents the true value, the circle represents the MAP estimate, and the cross with the horizontal line represents the mean and standard deviation. Right panel of each subfigure: comparison of the TL along the vertical array corresponding to the true values (dashed line) and to the MAP estimate (solid line).
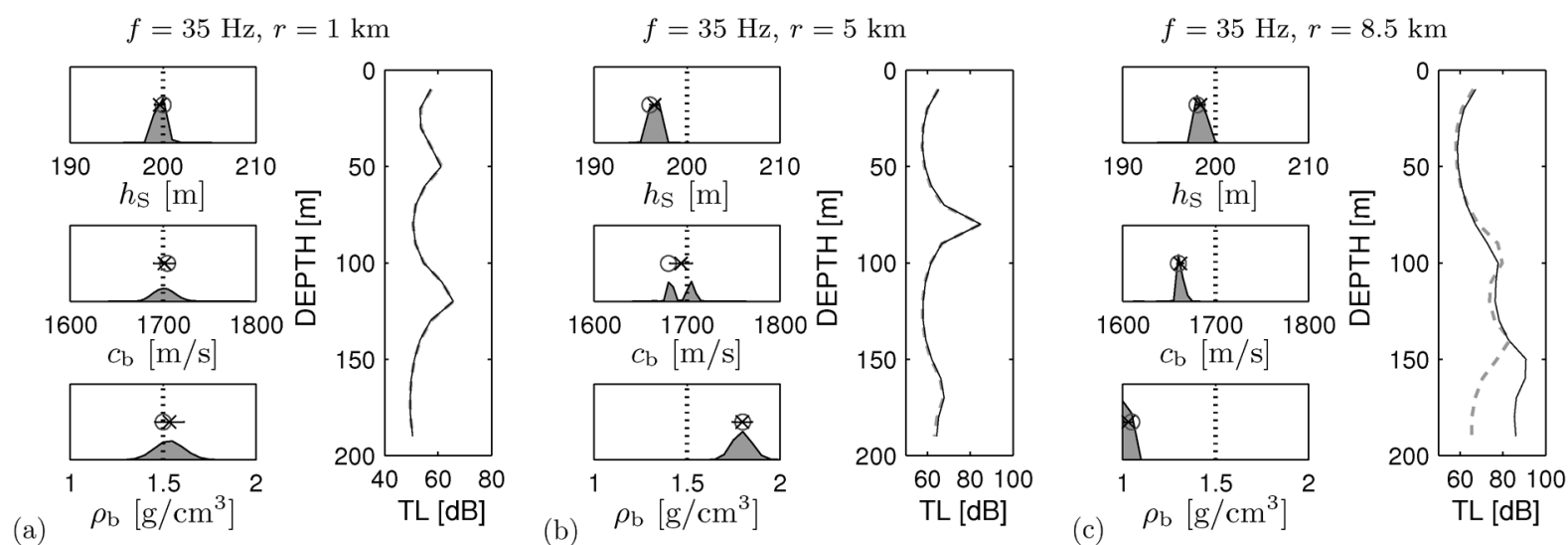

Fig. 11. Left panel of each subfigure: marginal posterior densities of $h_{\mathrm{S}}, c_{\mathrm{b}}$, and $\rho_{\mathrm{b}}$ for a 35-Hz frequency inversion assuming 2-D propagation along a vertical array located at (a) $1 \mathrm{~km}$, (b) $5 \mathrm{~km}$, and (c) $8.5 \mathrm{~km}$. The vertical dotted line represents the true value, the circle represents the MAP estimate, and the cross with the horizontal line represents the mean and standard deviation. Right panel of each subfigure: comparison of the TL along the vertical array corresponding to the true values (dashed line) and to the MAP estimate (solid line).

TABLE III

BARTLETT VALUES AT THE MAP ESTIMATE FOR INVERSION RANGES AND FREQUENCIES

\begin{tabular}{|c|c|c|}
\hline$r[\mathrm{~km}]$ & $f=25 \mathrm{~Hz}$ & $f=35 \mathrm{~Hz}$ \\
\hline 1.0 & 0.019 & 0.019 \\
\hline 5.0 & 0.019 & 0.019 \\
\hline 8.5 & 0.030 & 0.042 \\
\hline 13.5 & 0.033 & 0.045 \\
\hline 16.0 & 0.095 & 0.082 \\
\hline 19.0 & 0.134 & 0.148 \\
\hline
\end{tabular}

Note here that a 2-D model would predict single arrival contributions of each of the three modes at that range. The inversion process tunes the parameters to match the 2-D replica to the 3-D synthetic data, yielding parameter values (e.g., the MAP estimate) so that the 2-D computation, at the frequency of $25 \mathrm{~Hz}$, predicts the contribution of mode 1 alone at the receiving array as can be seen in the respective TL curve [solid line in Fig. 12(c)] which has no nodes as well.
Nonetheless, the 2-D computation is inherently unable to predict a second arrival of mode 1 at the receiving array. Consequently, the TL curves compare less favorably and a degradation as range increases is observed in the match between the data and the replica as reflected in the respective Bartlett values in Table III. Furthermore, the retrieved values for some parameters tend to be physically unacceptable. For instance, the bottom density at the frequency of $25 \mathrm{~Hz}$ for the ranges of 16 and $19 \mathrm{~km}$ tends to equal that of the water column. Accordingly, in this case of mismatch, we have strong indications that the 2-D-model-based inversion has failed. In both of these mismatch cases (i.e., with or without indications) fully 3-D modeling becomes necessary.

\section{CONCLUSION AND DisCUSSION}

In this work, we addressed the feasibility of inverting acoustic field data collected in shallow-water environments, like the continental shelf, based on 2-D modeling, i.e., ignoring out-of-plane propagation. The acoustic field in such environments may be affected by horizontal refraction leading to 3-D propagation effects and requiring fully 3-D modeling to be accounted for. Inverse problems in ocean acoustics are based on 2-D forward 

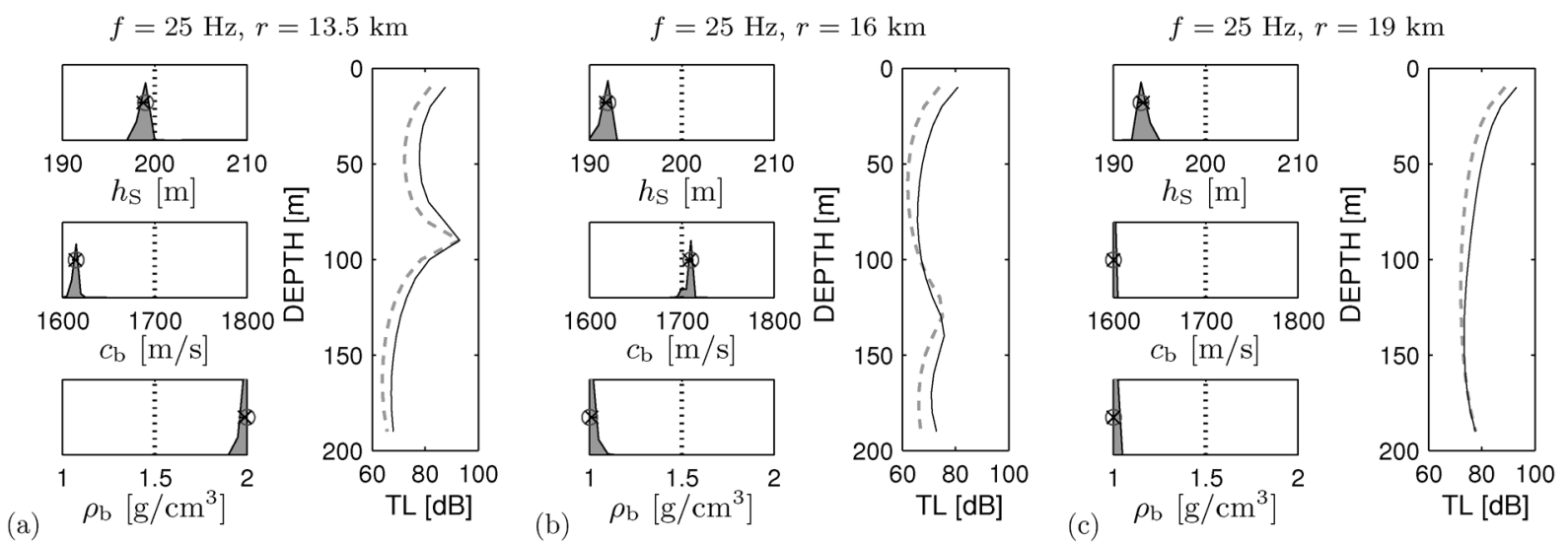

Fig. 12. Left panel of each subfigure: marginal posterior densities of $h_{\mathrm{S}}, c_{\mathrm{b}}$, and $\rho_{\mathrm{b}}$ for a 25-Hz frequency inversion assuming 2-D propagation along a vertical array located at (a) $13.5 \mathrm{~km}$, (b) $16 \mathrm{~km}$, and (c) $19 \mathrm{~km}$. The vertical dotted line represents the true value, the circle represents the MAP estimate, and the cross with the horizontal line represents the mean and standard deviation. Right panel of each subfigure: comparison of the TL along the vertical array corresponding to the true values (dashed line) and to the MAP estimate (solid line).
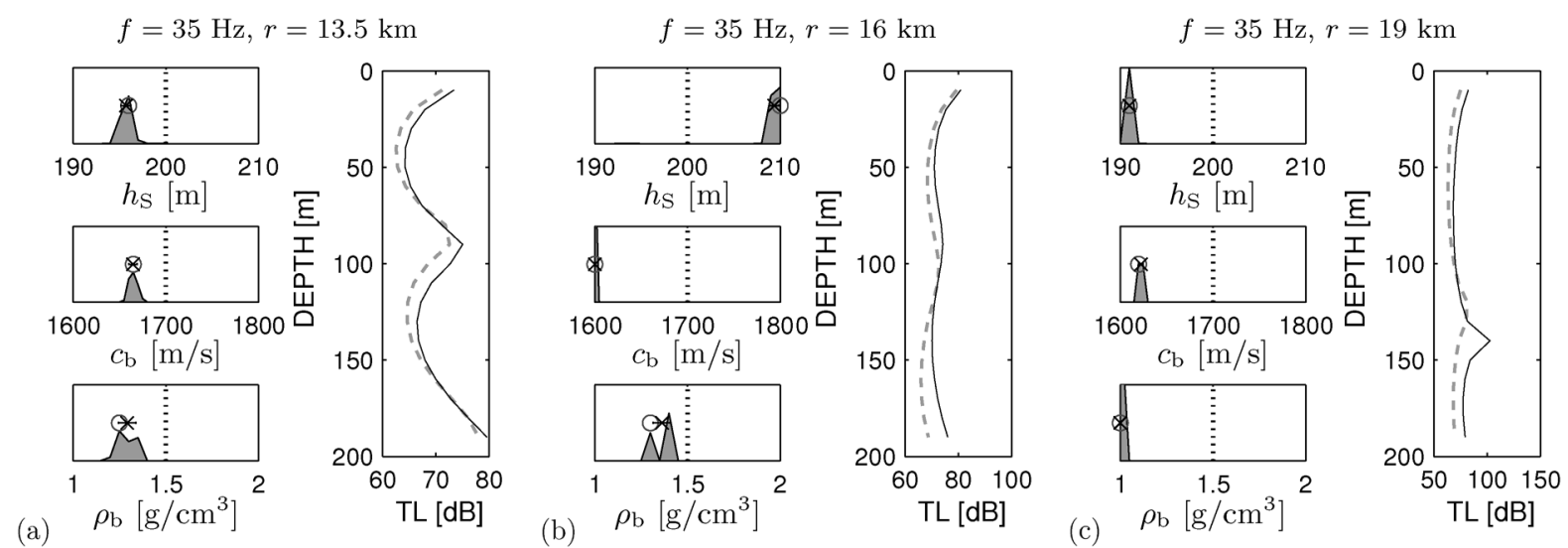

Fig. 13. Left panel of each subfigure: marginal posterior densities of $h_{\mathrm{S}}, c_{\mathrm{b}}$, and $\rho_{\mathrm{b}}$ for a $35-\mathrm{Hz}$ frequency inversion assuming 2-D propagation along a vertical array located at (a) $13.5 \mathrm{~km}$, (b) $16 \mathrm{~km}$, and (c) $19 \mathrm{~km}$. The vertical dotted line represents the true value, the circle represents the MAP estimate, and the cross with the horizontal line represents the mean and standard deviation. Right panel of each subfigure: comparison of the TL along the vertical array corresponding to the true values (dashed line) and to the MAP estimate (solid line).

models, as they represent a good compromise between speed and accuracy. However, it was not clear whether the 3-D effects can affect the performance and reliability of inversion procedures based on 2-D models, and, in particular, whether the retrieved parameters may turn out to be erroneous.

The present study was carried out on a well-established synthetic test case, namely, the 3-D ASA wedge benchmark representing a continental shelf environment, in which well-known 3-D effects occur. A typical matched-field inversion procedure was implemented based on the exhaustive search over the parameter space. The most important parameters with respect to the inversion were identified by means of a sensitivity analysis of the objective function. This preliminary analysis suggested that an inversion assuming 2-D propagation might succeed provided the array is located at relatively short ranges from the source. An inversion based on 2-D modeling was performed on synthetic data generated by a fully 3-D computation, simulating the acoustic pressure field on a VLA. The inversion results shown in this paper were obtained for two low frequencies and for several source/receiver distances in the across-slope direction. This approach demonstrated that the validity of the 2-D assumption depends on the specific propagation conditions at the array location. These in turn depend on the source/receiving array separation for a given environmental configuration. This can be summarized into three cases as follows.

1) Single arrivals of each mode initially excited at the source, which are not yet significantly affected by out-of-plane propagation, are received at the array. This is likely to occur at relatively short ranges from the source. In this case, the inversion process succeeds in matching the 2-D replica with the 3-D data and the correct parameter values are retrieved with satisfactory accuracy.

2) Single arrivals of each mode are still received at the array but now are significantly affected by out-of-plane propagation. In this case, the inversion process succeeds in matching the 2-D replica with the 3-D data and the parameter estimates are obtained with a good accuracy though they turn out to be biased with respect to the true parameter values. This is a typical case of mismatch.

3) Typical 3-D effects occur at the array location, like second mode arrivals and/or mode shadow zones. The 
inversion process barely matches the 2-D replica with the 3-D data and the estimated parameter values are erroneous and/or physically unacceptable.

Clearly, in case 1) the 2-D assumption is still valid whereas in cases 2) and 3) fully 3-D modeling is required. On the other hand, the importance of the effect of out-of-plane propagation, given a precise source/receiver separation, depends on the environmental configurations, mainly the slope and the water depth, and on the frequency. Specific regions where each of the above cases applies are thus problem dependent. Most importantly, in real-data applications, in which the parameters are not known before the inversion, the results should be interpreted with care as the distinction between cases 1) and 2) is not straightforward and the risk of mismatch is potentially important.

Let us conclude this paper with an attempt to interpret the results of a previous work already mentioned in the introduction. In [17], inversions of acoustic data collected in a continental shelf area near the Florida coast [18] were performed based on the 2-D assumption. The axis connecting the source and the vertical receiving array was nearly parallel to the shoreline and the water depth along that axis was considered nearly constant. The bottom in this area presented a weak slope towards the shoreline followed by an abrupt increase closer to the shoreline. A first inversion of the data collected on the array provided parameter estimates that were in poor agreement with available in situ measurements in that area. This was attributed to late arrivals in the data, corresponding to sound signals redirected by the steeper slope portion close to the shoreline. Numerical simulations using both a PE-based code and a ray code showed that these late arrivals were 3-D arrivals [19]. A 2-D-model-based inversion excluding these late arrivals in the data succeeded [27].

Let us now attempt to interpret this behavior in the light of our results. Considering the experimental conditions (slope, water depth, frequency, source-array separation, etc.; see [18]), we argue that it is likely that most of the early arrivals, having traveled through the weak slope area alone, have not been significantly affected by horizontal refraction and can thus be viewed as direct "2-D" arrivals. In other words, a 2-D-model-based inversion using the early arrivals alone is most likely to fall in case 1) (see above) for which the 2-D assumption is valid. On the other hand, the late arrivals traveled through the steeper slope area and were refracted downslope towards the receiving array: the late arrivals are fully 3-D arrivals. Consequently, a 2-D-model-based inversion including these late arrivals fails to retrieve the in situ measured parameter values. However, note here that this is a preliminary interpretation attempt and further investigation is needed.

\section{REFERENCES}

[1] C. H. Harrison, "Three-dimensional ray paths in basins, troughs, and near seamounts by use of ray invariants," J. Acoust. Soc. Amer., vol. 62, no. 6, pp. 1382-1388, 1977.

[2] C. H. Harrison, "Acoustic shadow zones in the horizontal plane," $J$. Acoust. Soc. Amer., vol. 65, no. 1, pp. 56-61, 1979.

[3] M. J. Buckingham, "Theory of three-dimensional acoustic propagation in a wedgelike ocean with a penetrable bottom," J. Acoust. Soc. Amer., vol. 82, no. 1, pp. 198-210, 1987.
[4] R. Doolittle, A. Tolstoy, and M. J. Buckingham, "Experimental confirmation of horizontal refraction of $\mathrm{CW}$ acoustic radiation from a point source in a wedge-shaped ocean environment," J. Acoust. Soc. Amer., vol. 83, no. 6, pp. 2117-2125, 1988.

[5] A. Tolstoy, "3-D propagation issues and models," J. Comput. Acoust., vol. 4, no. 3, pp. 243-271, 1996.

[6] A. Tolstoy, N. R. Chapman, and G. Brooke, "Workshop 97: Benchmarking for geoacoustic inversion in shallow water," J. Comput. Acoust., vol. 6, no. 1 \& 2, pp. 1-28, 1998.

[7] N. R. Chapman, S. Ching-Bing, D. King, and R. Evans, "Guest editorial special issue on geoacoustic inversion in range-dependent shallowwater environments," IEEE J. Ocean. Eng., vol. 28, no. 3, pp. 317-319, Jul. 2003.

[8] K. D. Heaney and J. J. Murray, "Measurements of three-dimensional propagation in a continental shelf environment," J. Acoust. Soc. Amer., vol. 125 , no. 3, pp. 1394-1402, 2009.

[9] D. B. Reeder, L. Y. S. Chiu, and C.-F. Chen, "Experimental evidence of horizontal refraction by nonlinear internal waves of elevation in shallow water in the south china sea: 3D versus Nx2D acoustic propagation modeling," J. Comput. Acoust., vol. 18, no. 3, pp. 267-278, 2010.

[10] L. Y. S. Chiu, A. Y. Y. Chang, C.-F. Chen, R.-C. Wei, Y.-J. Yang, and D. B. Reeder, "Three-dimensional acoustic simulation of an acoustic refraction by a nonlinear internal wave in a wedge bathymetry," $J$. Comput. Acoust., vol. 18, no. 3, pp. 279-296, 2010.

[11] K. B. Smith, "A three-dimensional propagation algorithm using finite azimuthal aperture," J. Acoust. Soc. Amer., vol. 106, no. 6, pp. 3231-3239, 1999.

[12] C. F. Chen, Y.-T. Lin, and D. Lee, "A three-dimensional azimuthal wide-angle model," J. Comput. Acoust., vol. 7, no. 4, pp. 269-288, 1999.

[13] E. K. Westwood, "Complex ray solutions to the 3-D wedge ASA benchmark problems," J. Acoust. Soc. Amer., vol. 109, no. 1, 2001, 2333:2aAOa6.

[14] J. A. Fawcett, "Modeling three-dimensional propagation in an oceanic wedge using parabolic equation methods," J. Acoust. Soc. Amer., vol. 93, no. 5, pp. 2627-2632, 1993.

[15] F. Sturm, "Numerical study of broadband sound pulse propagation in three-dimensional oceanic waveguides," J. Acoust. Soc. Amer., vol. 117, no. 3, pp. 1058-1079, 2005.

[16] F. Sturm, "On the use of higher-order azimuthal schemes in 3-D PE modeling," J. Acoust. Soc. Amer., vol. 113, no. 6, pp. 3134-3145, 2003.

[17] N. R. Chapman and Y. Jiang, "Geoacoustic inversion of broadband data from the Florida straits," in High Frequency Ocean Acoustics, M. B. Porter, M. Siderius, and W. A. Kuperman, Eds. New York: AIP Press, 2004, vol. 728, AIP Conference Proceedings, pp. 40-46.

[18] H. A. DeFerrari, N. J. Williams, and H. B. Nguyen, "Variability, coherence and predictability of shallow water acoustic propagation in the Straits of Florida," in Impact of Littoral Environmental Variablility on Acoustic Predictions and Sonar Performance, N. G. Pace and F. B. Jensen, Eds. Norwell, MA: Kluwer, 2002, pp. 245-254.

[19] F. Sturm, S. Ivansson, Y. Jiang, and N. R. Chapman, "Numerical investigation of out-of-plane sound propagation in a shallow water experiment," J. Acoust. Soc. Amer., vol. 124, no. 6, pp. EL341-EL346, 2008.

[20] F. B. Jensen and C. M. Ferla, "Numerical solutions of range-dependent benchmark problems in ocean acoustics," J. Acoust Soc. Amer., vol. 87, no. 4, pp. 1499-1510, 1990.

[21] P. Gerstoft and C. F. Mecklenbrauker, "Ocean acoustic inversion with estimation of a posteriori probability distributions," J. Acoust. Soc. Amer., vol. 104, no. 2, pp. 808-819, 1998.

[22] S. E. Dosso and G. Birdsall, "Quantifying uncertainties in geoacoustic inversion I: A fast Gibbs sampler approach," J. Acoust Soc. Amer., vol. 111 , no. 1, pp. 129-142, 2002.

[23] A. Tarantola, Inverse Problem Theory and Methods for Model Parameter Estimations. Philadelphia, PA: SIAM, 2005, ch. 1.

[24] M. K. Sen and P. L. Stoffa, "Bayesian inference, Gibbs' sampler and uncertainty estimation in geophysical inversion," Geophys. Prospecting, vol. 44, no. 2, pp. 313-350, Mar. 1996.

[25] C. F. Mecklenbrauker and P. Gerstoft, "Objective functions for ocean acoustic inversion derived by likelihood methods," J. Comput. Acoust., vol. 8, no. 2, pp. 259-270, 2000.

[26] G. L. D’Spain, J. J. Murray, W. S. Hodgkiss, N. O. Booth, and P. W. Schey, "Mirages in shallow water matched field processing," J. Acoust. Soc. Amer., vol. 105, no. 6, pp. 3245-3265, 1999.

[27] Y. Jiang, N. R. Chapman, and H. A. DeFerrari, "Geoacoustic inversion of broadband data by matched beam processing," J. Acoust Soc. Amer., vol. 119, no. 6, pp. 3707-3716, 2006. 


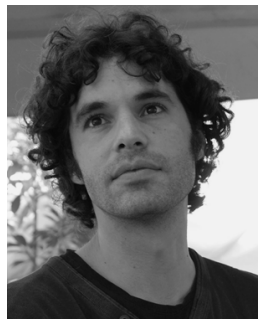

Alexios Korakas (M'08) received the B.Sc. degree in physics from the University of Crete, Heraklion, Greece, in 2005, the M.Sc. degree in acoustics from the University of Provence, Marseille, France, in 2006, and the Ph.D. degree in acoustics from the Ecole Centrale de Lyon, Ecully, France, in 2010.

From 2006 to 2010, he led his doctoral research at the Acoustics and Fluid Mechanics Laboratory (LMFA, UMR CNRS 5509). His research focused on both the experimental and numerical aspects of 3-D ocean acoustic propagation in shallow-water environments with respect to inversion. His research interests are in the area of inverse problems with applications to ocean acoustics.

Dr. Korakas is a Member of the Acoustical Society of America.

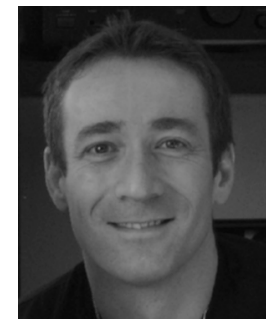

Frédéric Sturm was born in Toulon, France, in 1969. He received the M.Sc. degree in applied mathematics from the University of Aix-Marseille I, Marseille, France, in 1993 and the Ph.D. degree in acoustics from the University of Toulon, Toulon, France, in 1997.

After his doctoral dissertation, he was a Summer Research Assistant at the NATO SACLANT Undersea Research Centre, La Spezia, Italy. From 1998 to 1999 , he accomplished his postdoctoral research at the GESMA centre in Brest, France. Since 1999, he has been Assistant Professor at the INSA Engineering School, Lyon, France, where he teaches mathematics and numerical analysis in the internationally oriented section ASINSA. He is a coauthor of mathematics textbooks. He is involved in research activities of the Acoustics and Fluid Mechanics Laboratory (LMFA, UMR CNRS 5509-ECL-UCBL1-INSA Lyon). His current research interests include acoustic propagation and inversion problems in 3-D oceanic environments. His research combines both theoretical and experimental studies. 